\title{
How Massive Are the Superfluid Cores in the Crab and Vela Pulsars and Why Their Glitch-Events Are Accompanied with under and Overshootings?
}

\author{
A. A. Hujeirat ${ }^{1}$, R. Samtaney ${ }^{2}$ \\ ${ }^{1}$ IWR, Universität Heidelberg, Heidelberg, Germany \\ ${ }^{2}$ Applied Mathematics and Computational Science, CEMSE Division, KAUST, Thuwal, KSA \\ Email: AHujeirat@uni-hd.de,ravi.samtaney@kaust.edu.sa
}

How to cite this paper: Hujeirat, A. A. and Samtaney, R. (2020) How Massive Are the Superfluid Cores in the Crab and Vela Pulsars and Why Their Glitch-Events Are Accompanied with under and Overshootings? Journal of Modern Physics, 11, 395-406. https://doi.org/10.4236/jmp.2020.113025

Received: February 17, 2020

Accepted: March 13, 2020

Published: March 16, 2020

Copyright ( 2020 by author(s) and Scientific Research Publishing Inc. This work is licensed under the Creative Commons Attribution International License (CC BY 4.0).

http://creativecommons.org/licenses/by/4.0/

\begin{abstract}
The Crab and Vela are well-studied glitching pulsars and the data obtained so far should enable us to test the reliability of models of their internal structures. Very recently it was proposed that glitching pulsars are embedded in bimetric spacetime: their incompressible superfluid cores (SuSu-cores) are embedded in flat spacetime, whereas the ambient compressible and dissipative media are enclosed in Schwarzschild spacetime. In this letter we apply this model to the Crab and Vela pulsars and show that a newly born pulsar initially of $1.25 M_{\odot}$ and an embryonic SuSu-core of $0.029 M_{\odot}$ could evolve into a Crab-like pulsar after 1000 years and into a Vela-like pulsar 10,000 years later to finally fade away as an invisible dark energy object after roughly 10 Myr. Based thereon we infer that the Crab and the Vela pulsars should have $\mathrm{SuSu}$-cores of $0.15 M_{\odot}$ and $0.55 M_{\odot}$, respectively. Furthermore, the under- and overshootings phenomena observed to accompany the glitch events of the Vela pulsar are rather a common phenomenon of glitching pulsars that can be well-explained within the framework of bimetric spacetime.
\end{abstract}

\section{Keywords}

Relativity: Numerical, General, Black Hole Physics, Magnetars, Neutron Stars, Pulsars, Superfluidity, Superconductivity, Gluons, Quarks, Quantum Chromodynamics (QCD)

\section{Observational Constraints and Methodology}

The Crab and Vela pulsars are well-known and extensively studied pulsars (see 
[1]-[7] and the references therein). In Table 1 we summarize their basic observational data relevant for the present discussion. The SuSu-Scenario relies on solving the TOV equation in combination with the equations of torque balance between the incompressible superfluid cores, whose dynamics obey the Onsager-Feymann equation, and an overlying shell of compressible and dissipative matter (see Sec. 2 and Eq. 10 in [8]).

In [9] these equations were solved at the background of a bimetric space-time (see Figure 1). Unlike the original model [8], in which the spin-down of the Su$\mathrm{Su}$-core is set to follow a priori given sequence of values $\left\{\Omega_{c}^{n}\right\}$, in the present work however, the SuSu-core is set to undergo an abrupt spin-down, if the difference between its Eigen rotation and that of the ambient medium surpasses a critical value $\left\{\Delta \Omega_{c r}^{n}\right\}$, i.e. if $\Delta \Omega_{c-a m}^{n}=\Omega_{c}^{n}-\Omega_{a m}^{n} \geq \Delta \Omega_{c r}^{n}$, where " $n$ " and "am" refer to the order of the elements in the relevant sequence and to the ambient medium, respectively. This approach is more consistent than the former, as the elements of $\Delta \Omega_{c r}^{n}$ are determined here through the rate of loss of rotational energy of the entire star and that these should overlap the current values observed in the Crab and Vela pulsars.

The strategy of obtaining the optimal values here relies on using a global iterative solution procedure that takes the following constraints into account (see also Table 1$)$ :

1) The elements of the sequence $\left\{\frac{\Delta \Omega_{g}}{\Omega}\right\}^{n}$ must fulfill the three conditions:

- $\left\{\frac{\Delta \Omega_{g}}{\Omega}\right\}^{n} \underset{n \rightarrow 0}{\longrightarrow} 0$, which means that the media in both the core and in the surrounding shell must have identical rotational frequency initially.

- $\left.\frac{\Delta \Omega_{g}}{\Omega}\right|_{n=N_{0}}=\left.\frac{\Delta \Omega_{g}}{\Omega}\right|_{\text {Crab }}=4 \times 10^{-9}$ and $\left.\frac{\Delta \Omega_{g}}{\Omega}\right|_{n=N_{1}}=\left.\frac{\Delta \Omega_{g}}{\Omega}\right|_{\text {Vela }}=2.33 \times 10^{-6}$, i.e. the elements number $N_{0}$ and $N_{1}\left(\gg N_{0}\right)$ of the sequence $\left\{\frac{\Delta \Omega_{g}}{\Omega}\right\}^{n}$ must be identical to the observed values of the Crab and to the Vela pulsars, respectively.

Table 1. A list of the main observational data of the Crab and Vela pulsars relevant for the present study (see [2]-[7] and the references therein).

\begin{tabular}{ccc}
\hline & Crab & Vela \\
\hline Mass $\left(M_{\odot}\right)$ & 1.4 & 1.8 \\
Age $(\mathrm{kyr})$ & 1.24 & 11.3 \\
$B\left(10^{12} \mathrm{G}\right)$ & 4.875 & 4.35 \\
$\Omega\left(\mathrm{s}^{-1}\right)$ & 200 & 70 \\
$\Delta \Omega_{g} / \Omega$ & $4 \times 10^{-9}$ & $2.338 \times 10^{-6}$ \\
$\Delta t_{g}(\mathrm{yr})$ & 1.6 & 2.5 \\
\hline
\end{tabular}




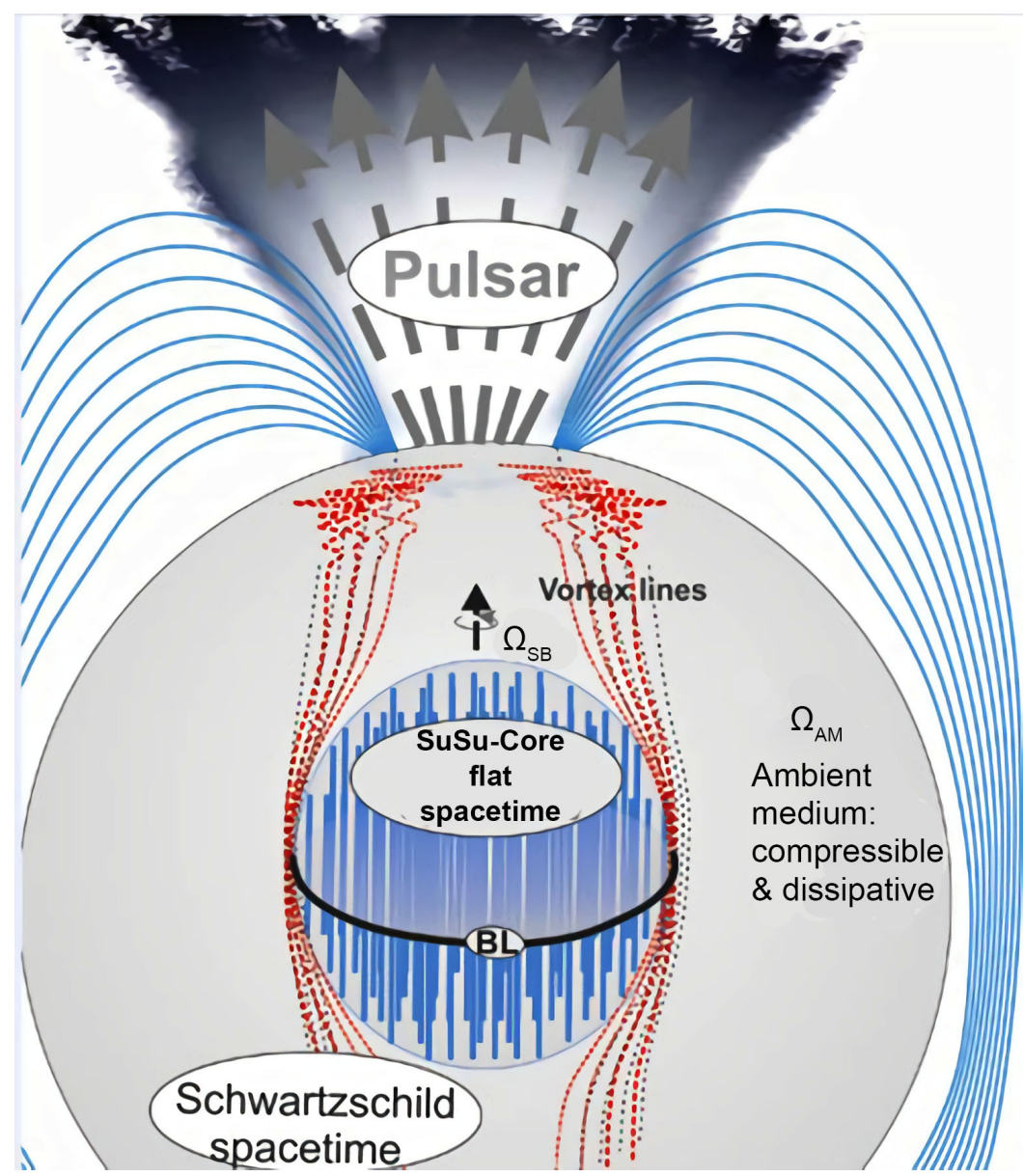

Figure 1. A schematic description of the bimetric spacetime inside glitching pulsars: The incompressible superfluid core is embedded in a Minkowski spacetime whereas the ambient media are enclosed in Schwarzschild spacetime.

- $\left\{\frac{\Delta \Omega_{g}}{\Omega}\right\}^{n} \underset{n \rightarrow \infty}{\longrightarrow} \alpha_{\infty}<\infty$, i.e. the sequence must converge to a finite value. Moreover, our test calculations have shown that for $t \gg t_{\text {Vela }}$, $\frac{\partial}{\partial t}\left(\left\{\frac{\Delta \Omega_{g}}{\Omega}\right\}^{n}\right)<0$ as otherwise the magnetic field would fail to spin-down the crust and therefore to surpass $\Delta \Omega_{c r}$ required for triggering a prompt spin-down of the core into the next lower energy state.

Indeed, one possible sequence which fulfills the above-mentioned constraints, though it might not be unique, is shown in Figure 2.

2) The initial conditions used here are $\Omega_{0}=\Omega(t=0)=1440 \mathrm{~Hz}$ (see [10] and the references therein). Here both the core and the ambient medium are set to initially rotate with the same frequency, i.e. $\Omega_{c}^{n}=\Omega_{a m}^{n}(t=0)=\Omega_{0}$. The initial total mass of the pulsars and that of the core are taken to be: $M_{c}=\epsilon_{0} M_{\odot}$ and $M_{0}=M(t=0)=\epsilon_{1} M_{\odot}$, respectively, where $\epsilon_{0}$ and $\epsilon_{1}$ are parameters whose values are determined through a global iteration procedure. The initial magnetic field strength is taken to be $B(t=0)=10^{13}$ Gauss. 


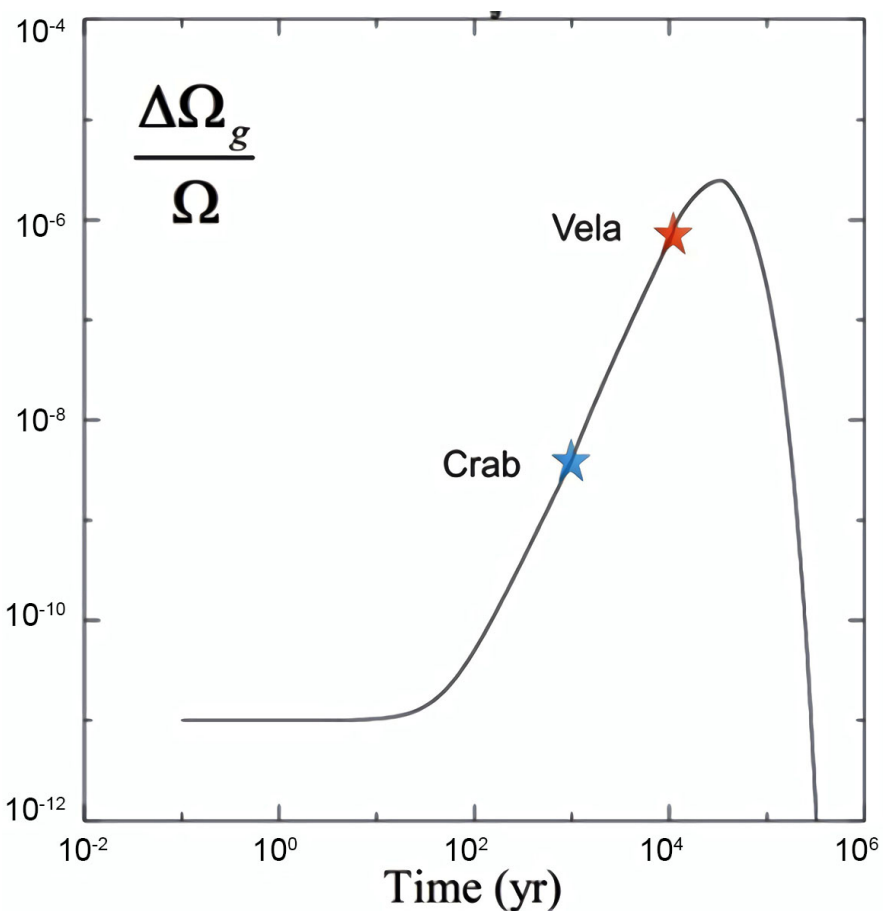

Figure 2. The elements of the quantum sequence $\Delta \Omega_{g}^{n} / \Omega$ are shown as function of time in year units. Both the core and the ambient medium are set to rotate with the same frequency $1440 \mathrm{~s}^{-1}$ initially. As the pulsar cools down, $\Delta \Omega_{g}^{n} / \Omega$ starts increasing to reach 4 $\times 10^{-9}$ after approximately $1000 \mathrm{yr}$, (which corresponds to the Crab phase/blue-star) and $8.15 \times 10^{-7}$ after $11,000 \mathrm{yr}$ (which corresponds to Vela phase/red-star). $\Delta \Omega_{g}^{n} / \Omega$ here is measured in units of $\Omega=200 / \mathrm{s}$.

3) The elements of the sequence $\left\{\Omega_{a m}\right\}^{n}$ are obtained through the energy balance equation:

$$
\frac{\mathrm{d}}{\mathrm{d} t}\left(\frac{1}{\Omega_{a m}^{2}}\right)=-\alpha_{E M} \frac{B^{2}}{I_{a m}}
$$

where $I_{a m}$ is the inertia of the ambient compressible dissipative medium, which, due to the increase of the SuSu-core, must decrease on the cosmic time and $\alpha_{E M}=4.9 \times 10^{-4}$ is a non-dimensional constant.

Thus, the time-evolution of the core's rotational frequency proceeds as follows: for a given $\Omega_{c}^{n}$, the ambient medium is set to decrease its frequency continuously with time through the emission magnetic dipole radiation. This implies that the difference $\Delta \Omega_{c-a m}$ should increase with time until $\Delta \Omega_{c-a m}$ has surpassed the critical value $\geq \Delta \Omega_{c r}$, In this case three events are expected to occur promptly:

- The rigid-body rotating core changes abruptly its rotational state from $E_{c}^{n, r o t}=\frac{1}{2} I_{c}^{n}\left(\Omega_{c}^{n}\right)^{2}$ into the next the quantum-mechanically permitted lower energy state: $E_{c}^{n+1, r o t}=\frac{1}{2} I_{c}^{n+1}\left(\Omega_{c}^{n+1}\right)^{2}$. This process is associated with ejection of a certain number of vortices into the boundary layer $(\mathrm{BL})$ between the core 
and the overlying dissipative medium.

- The ejected vortices by the core are then absorbed by the differentially rotating dissipative medium and re-distributed viscously. Hence the medium in the BL would experience the prompt spin-up: $\Omega_{a m}^{n} \rightarrow \Omega_{a m}^{n}+\Delta \Omega_{g}^{n}$, where $\Delta \Omega_{g}^{n}$ is deduced from the sequence $\left\{\frac{\Delta \Omega_{g}}{\Omega}\right\}^{n}$.

- The radius of the core is set to increases as dictated by the Onsager-feynmann equation:

$$
\oint V \cdot \mathrm{d} \ell=\frac{2 \pi \hbar}{m} N,
$$

where $V, \ell, \hbar, m, N$ denote the velocity vector, the vector of line-element, the reduced Planck constant, mass of the superfluid particle pair and the number of vortices, respectively (see [11] for further details). Imposing zero-torque condition on the incompressible SuSu-core, i.e., $\frac{\mathrm{d}}{\mathrm{d} t}(\Omega I)_{c}=0$. We then obtain the following recursive relation:

$$
\begin{aligned}
& (\Omega S)_{c}^{n+1}=(\Omega S)_{c}^{n} \\
& \Rightarrow S^{n+1}=\left(\frac{\Omega_{c}^{n}}{\Omega_{c}^{n+1}}\right) S^{n}
\end{aligned}
$$

where $S_{c}^{n} \doteq \pi\left(R_{c}^{n}\right)^{2}$ and $R_{c}^{n}$ correspond to the cross-sectional area of the Su$\mathrm{Su}$-core and to the corresponding radius, respectively. The increase in the dimension of the core implies that the matter in the geometrically thin boundary layer between the SuSu-core and the ambient medium should undergo a crossover phase transition into an incompressible superfluid, whose total energy density saturates around the critical value $\rho_{c r} \approx 6 \rho_{0}$ (see [12] and the references therin). The growth of the core proceeds on the cosmic time scale and ends when the pulsar has metamorphosed entirely into a maximally compact invisible dark energy object and therefore becomes observationally indistinguishable from a stellar black hole.

\section{Solution Procedure \& Results}

The set of equations consists of the TOV equation for modeling the compressible dissipative matter in the shell overlaying the incompressible gluon-quark superfluid core, whereas the latter is set to obey the zero-torque condition and to dynamically evolve according to the Onsager-Feymann equation (for further details see Sec. 2 and Eq. 10 in [8]).

The global iteration loop is designed here to find the optimal values of the parameters: $\alpha_{0}, \alpha_{1}$, the elements of the sequence $\Delta \Omega_{c r}^{n}$ and the decay rate of the magnetic field. These values should fulfill the initial and final conditions, the currently observed values of the time passages between two successive glitch-events $\Delta t_{g}$ both of the Crab and the Vela pulsars, the current observed values of their magnetic fields masses. 
Indeed, our intensive computations reveal that optimal fitting may be achieved for $M_{c}(t=0) \approx 0.029 M_{\odot}$, a sequence of $\Delta \Omega_{g}^{n}$, whose elements are shown in Figure 2. In Figure 3, Figure 4 the optimal values of $\Delta \Omega_{c r}^{n}$ and $\Delta \Omega_{g}^{n}$ are shown versus cosmic time, whereas in Figure 5 we show the time-development of the rotational frequencies of the core, the ambient medium and of the magnetic field during the first 10 to 100 years after the birth of the pulsar. The long-term evolution of the magnetic field and the growing mass of the core and of the entire object are shown in Figure 6 and Figure 7. Here the mass of the pulsar's core grows with time to reach $0.15 M_{\odot}$ after 1000 years and reaches $0.55 M_{\odot}$ after 11,000 years; hence reproducing the exact total masses of $1.4 M_{\odot}$ for the Crab and $1.8 M_{\odot}$ for the Vela pulsars as revealed from observations. The relative ratio of inertia of both cores reads $I^{\text {Crab }} / I^{\text {Vela }} \approx 3 / 20$.

Due to the incompressible, superfluid and supreconducting character of the core, the evolution of the magnetic field is solely connected to the dynamics of the ambient compressible and dissipative matter in the shell as well as to its dimensions (see [13] for further details on the physical aspects of compressibility of fluid flows). As the mass and dimension of the core grow with time, the surrounding shell must shrink. In this case, conservation of the magnetic flux should strengthen the magnetic field intensity. This interplay between the loss of magnetic energy due to loss of rotational energy and enhancement by conservation of magnetic flux in combination with dynamo action and other mechanisms,

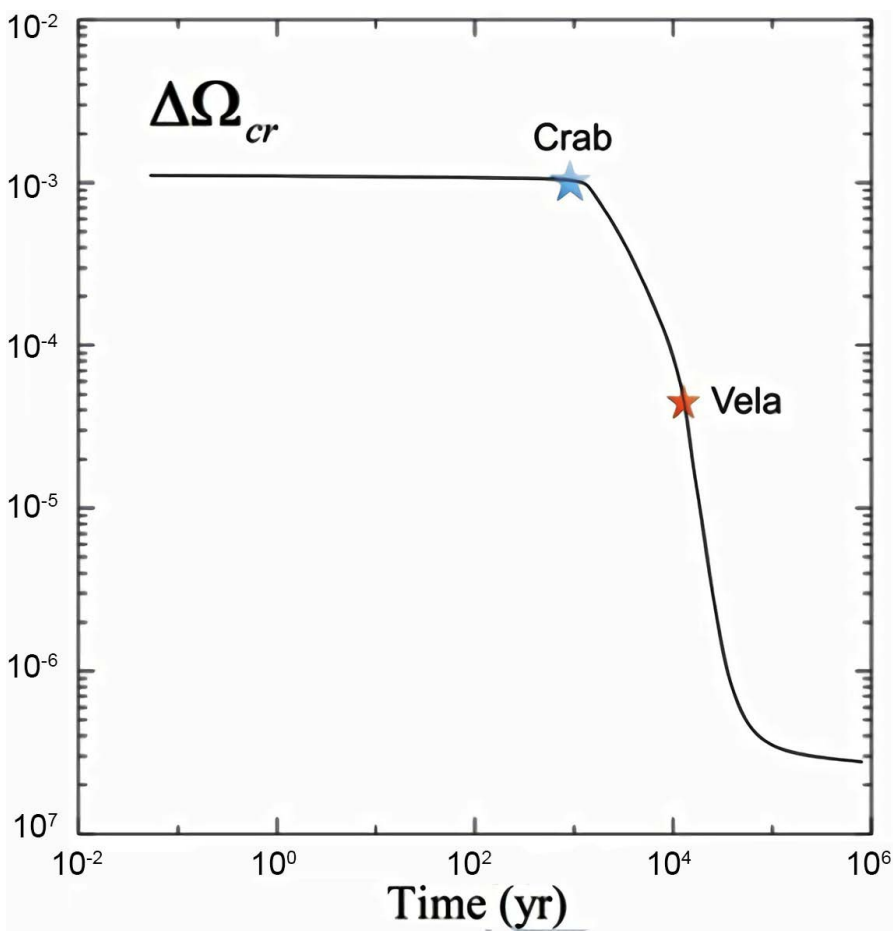

Figure 3. The elements of the sequence $\Delta \Omega_{c r}^{n}$ versus cosmic time. Each element corresponds to the critical difference between the rotational of frequency of the core and that of the ambient medium, beyond which the core undergoes a prompt spin-down to the next lower energy state. 


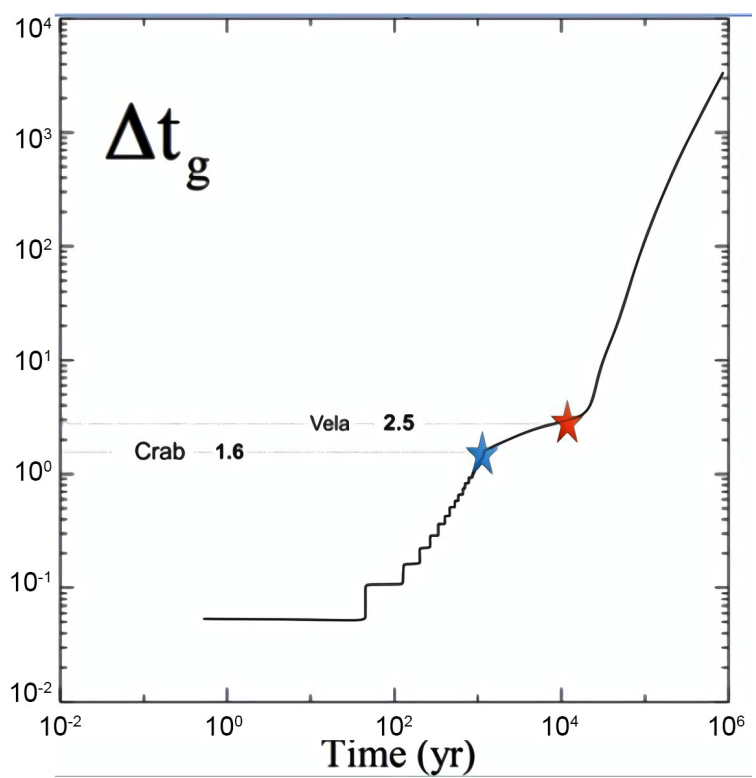

Figure 4. The elements of the sequence $\Delta t_{g}$ versus cosmic time. Each element corresponds to the time passage between two successive glitch events. The actual values that correspond to the Crab and Vela pulsars are shown in blue and red stars. These time passages increase dramatically with time due to the decay of both magnetic field intensity and rotational energy.

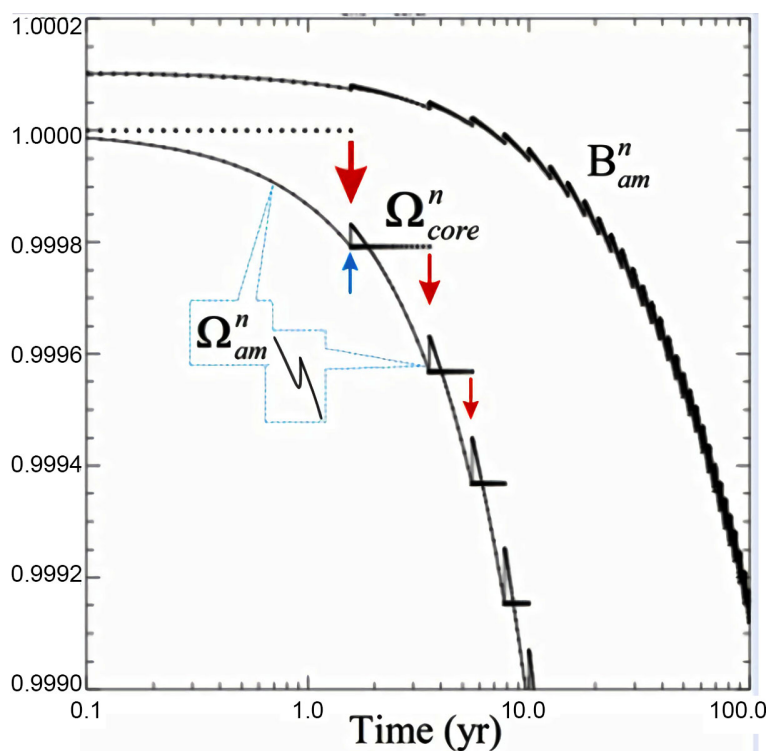

Figure 5. The elements of the sequences $\Omega_{c}^{n}, \Omega_{a m}^{n}$ and $B_{a m}^{n}$ that corresponds to the rotational frequencies of the core, the ambient medium and of the magnetic field during the first 10 years. During a passage of time between two successive glitches, the core rotates rigidly with a constant frequency $\Omega_{c}^{n}=\Omega_{\text {core }}^{n}$ (dotted line), whereas the ambient medium spin-down in a continuous manner (solid line). During the glitch, the core spin-down abruptly, triggering a prompt spin-up of the ambient medium in the boundary layer between the rigid-body rotating core and the overlying differentially rotating medium. The enhanced spin-up of the ambient medium in combination with the decreasing volume enclosing this matter gives rise to magnetic field $B_{a m}$ which evolves in a similar discrete manner (dash-dot). 


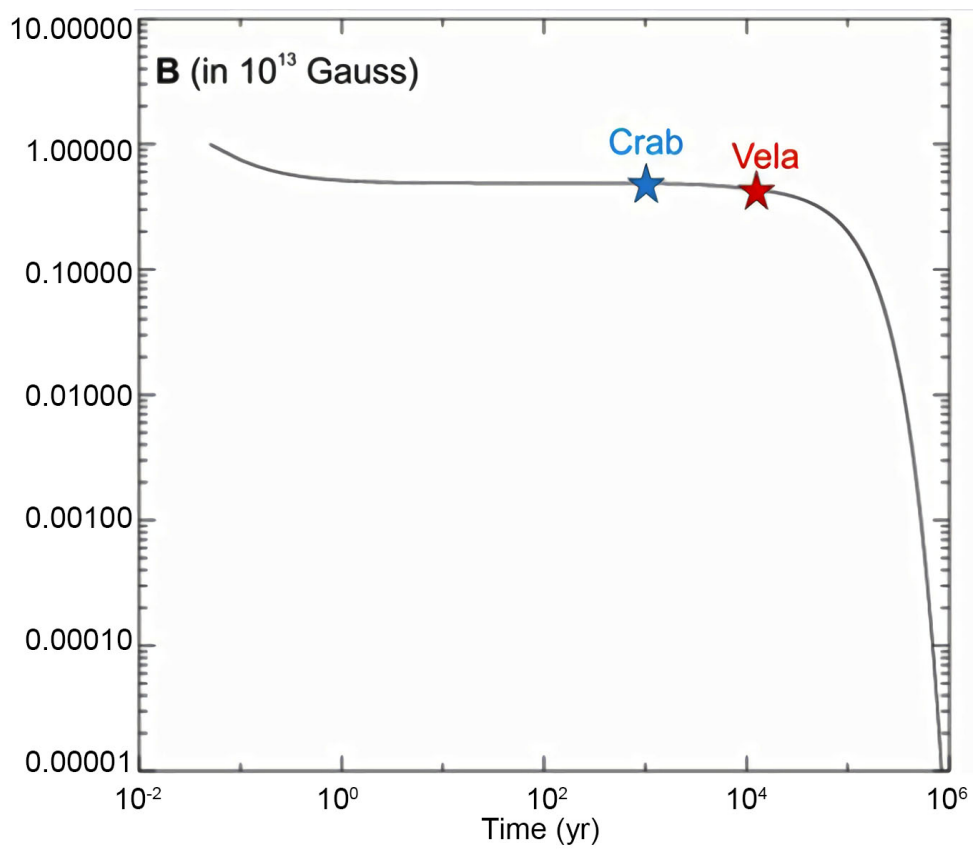

Figure 6. The cosmic evolution of the magnetic field-B, of a newly born pulsar in units of $10^{13} \mathrm{G}$. The superimposed blue and red stars correspond to the current B of the Crab and Vela pulsars.

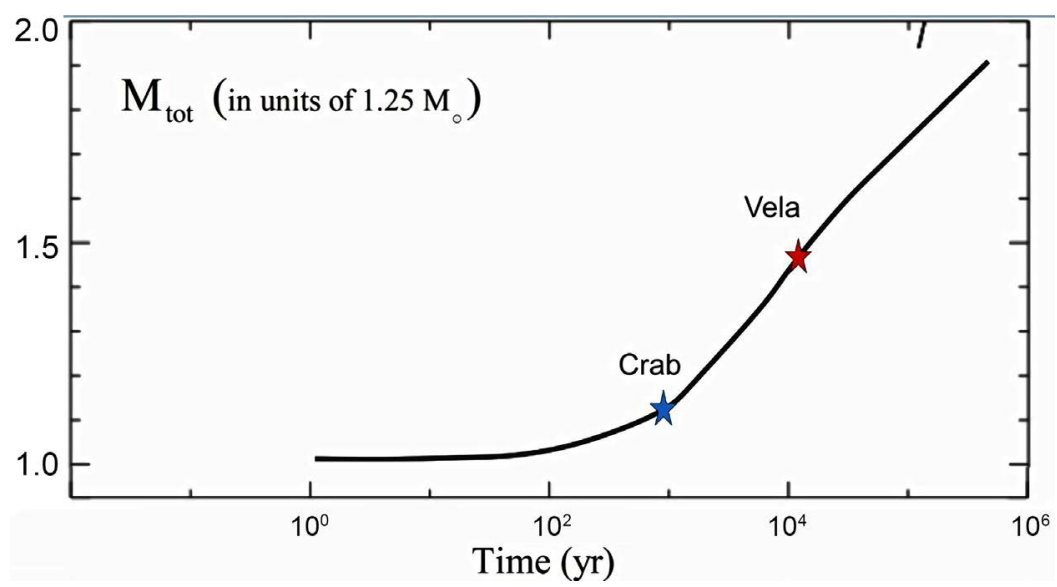

Figure 7. The mass growth of a newly born pulsar having initially $M_{0}=1.25 M_{\odot}$ and an embryonic SuSu-core of $0.029 M_{\odot}$. After approximately $1000 \mathrm{yr}$ the pulsar recover the mass of the $\operatorname{Crab}\left(M_{0}=1.4 M_{\odot}\right)$ and $10,000 \mathrm{yr}$ later $M_{0}=1.8 M_{\odot}$ that corresponds to the mass of the Vela. At the end of the luminous life time, which lasts for approximately 10 million years, the pulsar enters the dark phase with a total mass of $2.5 M_{\odot}$, which corresponds to a maximally compact invisible dark energy object. The plotted mass here is in units of $M_{0}$.

may clarify the very weak decay of magnetic field as pulsars evolve from the Crab to the Vela phase. Mathematically, let the magnetic energy in a shell of a newly born pulsar be:

$$
E_{M}=\int \frac{B^{2}}{8 \pi} \mathrm{d} v \sim \frac{B^{2}}{6}\left(R_{\star}^{3}-R_{c}^{3}\right),
$$


where $R_{\star}$ denotes the pulsar's radius. Assuming $E_{M}$ to roughly decay as the rotational energy $E_{\Omega}$, then we obtain:

$$
B^{-}=\alpha_{\Omega} \Omega_{a m}^{2} M_{a m}^{1 / 2} \sqrt{\frac{R_{\star}^{2}-R_{c}^{2}}{R_{\star}^{3}-R_{c}^{3}}},
$$

where $\alpha_{\Omega}$ is constant coefficient.

On the other hand, dynamo action in combination with magnetic flux conservation and other enhancement mechanisms would contribute positively to the magnetic field, that can, for simplicity absorbed in the term: $B^{+}=\alpha_{B} /\left(R_{\star}^{2}-R_{c}^{2}\right)$. The coefficient $\alpha_{B}$ is set to ensure that the magnetic field remains in the very sub-equipartition regime. Hence the interplay between magnetic loss and enhancement would yield an effective magnetic field that evolves according to:

$$
B_{\text {tot }}=\frac{\alpha_{B}}{R_{\star}^{2}-R_{c}^{2}}-\alpha_{\Omega} \Omega_{a m}^{2} M_{a m}^{1 / 2} \sqrt{\frac{R_{\star}^{2}-R_{c}^{2}}{R_{\star}^{3}-R_{c}^{3}}} .
$$

Consequently, our model predicts that the decreasing volume of the shell enclosing the ambient medium in combination with dynamo action in the boundary layer could potentially be the mechanism that keeps the decay of magnetic fields in pulsars extremely weak.

In fact our model predicts the glitch activity of a newly born pulsar, which evolves into a Crab phase, followed by a Vela phase and finally by an invisible phase, to be approximately two orders of magnitude larger than it was estimated by other models (see Figure 8 to be compared to [1] [5]). According to our

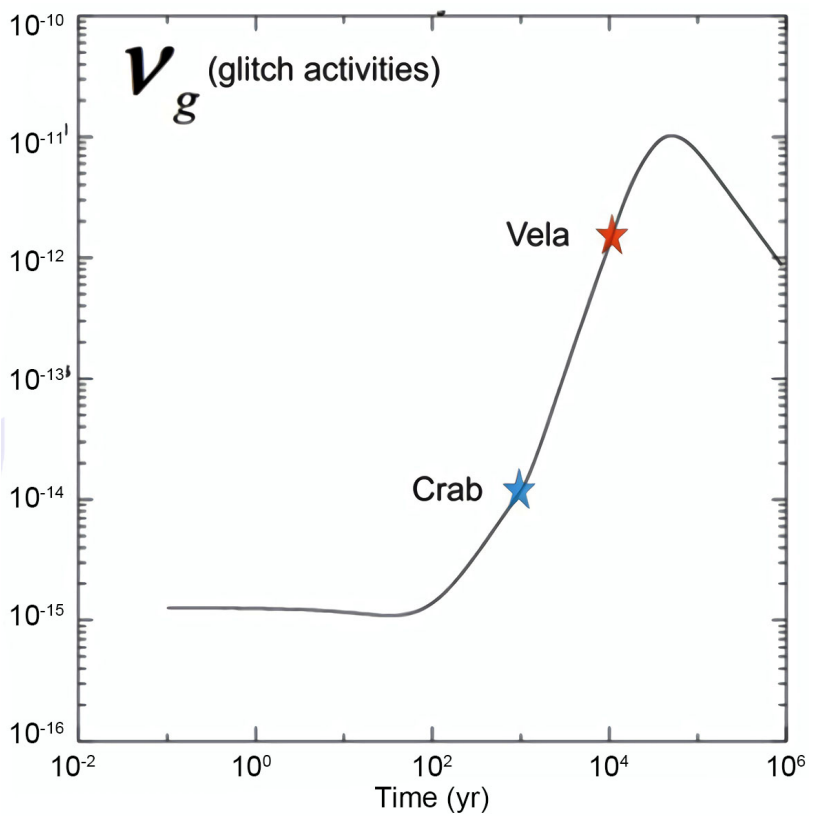

Figure 8. The glitch activity of a newly born pulsar versus cosmic time. In the very early times, the pulsar underwent millions of glitches, though the total ejected rotational energy was relatively very low. These activities start to be significant as the pulsar ages and become maximally effective between 100 and 60,000 years, followed by a decreasing phase, during which time-passages between successive glitches become increasingly longer. 
model pulsar may undergo millions or up to billions of glitches during their luminous life time with passages of time between two successive glitch events that range from nanoseconds in the very early time up to hundreds or even thousands of years toward the end of their luminous life times (see Figure 4). The vast difference in the evolution of glitch activity between the two approaches here may be attributed to the strong non-uniformity of time-duration between glitch events.

Moreover, the model also predicts the occurrence of under- and overshootings that have been observed to accompany the glitch events in the Vela pulsar (see [14] and the references therein). In the case of the Vela, when the core expels certain number of vortices and moves to the next lower energy state, the enhanced rotational energy of the matter in the BL amplifies the magnetic field strength. Due to the non-locality of magnetic fields ${ }^{1}$, this enhancement is communicated to the crust via Alfven waves, $V_{A}$, whereas the excess of rotational energy is communicated via shear viscosity with an effective propagational velocity $V_{\text {vis }}$. As these two speeds are generally different with $V_{A}>V_{\text {vis }}$ in most cases, the time-delay in the arrival of communication enforces the crust to react differently. Specifically, the arrival of magnetic enhancement prior to the rotational one leaves the crust subject to an enhanced magnetic braking and therefore to a stronger reduction of its rotational frequency (see the top panel of Figure 9).

Indeed, in the case of Vela, the propagational speed of Alfven waves may be esitmated to be of order $V_{A} \sim B / \sqrt{\rho} \approx 10^{8} \mathrm{~cm} / \mathrm{s}$. Hence the enhanced MFs in the BL would be communicate to the crust within

$\delta \tau_{M F}=\Delta R / V_{A}=\left(R_{\star}-R_{c}\right) / V_{A} \approx 10^{-2} \mathrm{~s}$. On the other hand, supplying the crust with rotational energy would proceed on the viscous time scale, which is estimated to be: $\delta \tau_{\text {vis }}=(\Delta R)^{2} / v_{\text {vis }}$ (see [15] and the references therein). Under normal astrophysical conditions we may safely assume that $V_{\text {vis }} / V_{A}=\alpha_{2} \ll 1$ and that the length scale, $\ell_{\text {vis }}$, over which viscous interaction occurs, $\ell_{\text {vis }}$ is much smaller than the width of the shell $\Delta R$, or equevalently $\ell_{\text {vis }}=\alpha_{2} \Delta R$, with $\alpha_{2}=1$. Therefore $\delta \tau_{\text {Vis }}=\Delta R /\left(\alpha_{1} \alpha_{2} V_{A}\right) \approx 10^{-2} /\left(\alpha_{1} \alpha_{2}\right) \approx 1 \mathrm{~s}$, where we reasonably set $\alpha_{1}=\alpha_{2}=0.1$. Consequently, the observed undershooting most likely results due to the time delay of the arrival of communications via magnetic fields and viscous torque, which amounts to $\delta \tau_{v i s} / \delta \tau_{M F}=1 /\left(\alpha_{1} \alpha_{2}\right) \approx 100$. On the other hand, the observed overshooting can be attributed to the case in which the viscous front transporting rotational energy from the BL outwards has reached the crust. As $\delta \tau_{M F} \ll \delta \tau_{v i s}$, at the end of $\delta \tau_{v i s}$, the magnetic field intensity in the $\mathrm{BL}$ should have returned to values comparable or even lower than prior to the glitch event.

Moreover, the observed order in which undershooting followed by overshooting is an indication for a time-delay in the arrival of communication resulting from $V_{A}>V_{\text {vis }}$ and from the significant difference of the locations of the $\mathrm{BL}$ and the crust. This order is expected to reverse if $V_{A}<V_{v i s}$.

${ }^{*}$ I.e. In the absence of magnetic monopoles. 


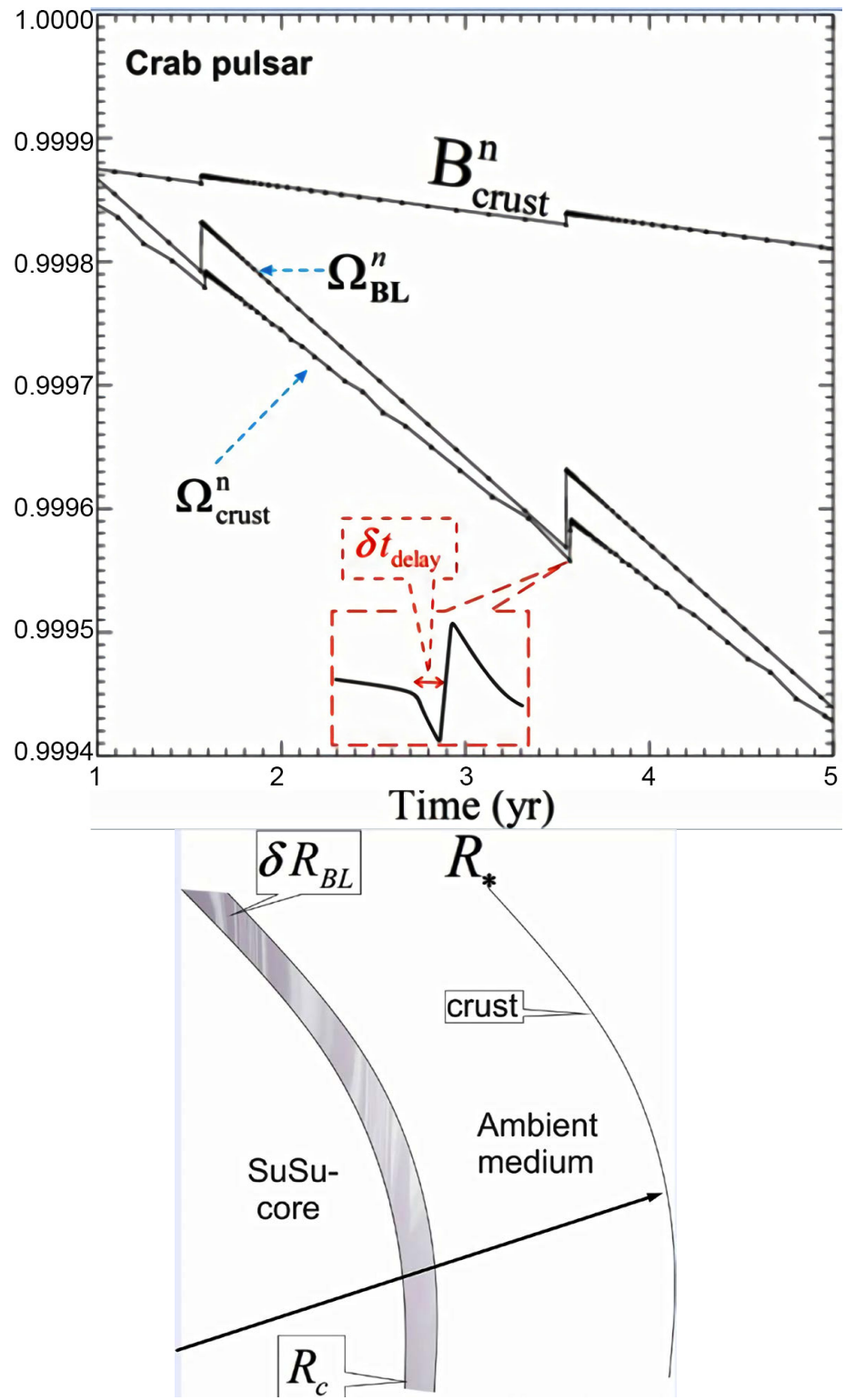

Figure 9. Under and overshooting of $\Omega_{a m}$ shortly before and immediately after a glitch event (top Figure). At a given instant of time, $t$, the rotational frequency of the matter in the BL, $\Omega_{a m}$, differs from that of the crust $\Omega_{\text {crust }}$. Due to their different locations (see lower panel), the response of the crust to the dynamical changes of matter in the BL depends strongly on the speed of communication via magnetic fields (e.g. Alfven waves) and shear viscosity, which, under most astrophysical conditions, are considered to be different.

In fact, the under- and overshooting here may indicate that MFs are insensitive to the momentary rotational frequency of the crust, but rather to the activity and dynamics of the matter in the BL.

Extending this analysis to both the Crab and Vela pulsars, the relative time-delays is expected to be: $\delta \tau_{\text {vis }}^{\text {Crab }} / \delta \tau_{\text {vis }}^{\text {Vela }} \sim\left(\Delta R^{\text {Crab }} / \Delta R^{\text {Vela }}\right)^{2} \approx 3.4$ or equivalently, the undershooting in the case of the Crab is expected to last $3.4 \mathrm{sec}$ comapred to one second in the Vela case. 
Finally, although the physics is entirely different, the situation here is strikingly similar to action of the solar dynamo, which is considered to be located in the so-called tachcline between the rigid-body rotating core and the overlying convection zone [16].

\section{Acknowledgements}

The calculations have been carried out using the computer cluster of the IWR, University of Heidelberg. RS acknowledges the use of KAUS baseline research funds

\section{Conflicts of Interest}

The authors declare no conflicts of interest regarding the publication of this paper.

\section{References}

[1] Roy, J., Yashwant Gupta, Y. and Lewandowski, W. (2012) MNRAS, 424, 2213-2221.

[2] Yu, M., Manchester, R.N., Hobbs, G., et al. (2013) MNRAS, 429, 688-724.

[3] Yuan, J., Kou, F. and Wang, N. (2019) AIP Conference Proceedings, 2127, Article ID: 020004.

[4] Eya, I.O. and Urama, J.O. (2014) International Journal of Astrophysics and Space Science, 2, 16

[5] Espinoza, C.M., Lyne, A.G., Stappers, B.W. and Kramer, C. (2011) MNRAS, 414, 1679. https://doi.org/10.1111/j.1365-2966.2011.18503.x

[6] Fuentes, J.R., Espinoza, C.M., Reisenegger, A., et al. (2017) $A \& A, 608$, A131. https://doi.org/10.1051/0004-6361/201731519

[7] Ozel, F. and Freire, P. (2016) Annual Review of Astronomy and Astrophysics, 54, 401. https://doi.org/10.1146/annurev-astro-081915-023322

[8] Hujeirat, A.A. (2018) Journal of Modern Physics, 9, 4.

[9] Hujeirat, A.A. and Samtaney, R. (2019) Journal of Modern Physics, 9, 4.

[10] Haensel, P., Lasota, J.P. and Zdunik, J.L. (1999) $A \& A, \mathbf{3 4 4}, 151$.

[11] Hujeirat, A.A. (2018) Journal of Modern Physics, 9, 51-69. https://doi.org/10.4236/jmp.2018.91004

[12] Hujeirat, A.A. (2018) Journal of Modern Physics, 9, 4.

[13] Hujeirat, A.A. and Thielemann, F.-K. (2009) MNRAS, 400, 903. https://doi.org/10.1111/j.1365-2966.2009.15498.x

[14] Ashton, G., Lasky, P.D., Graber, V. and Palfreyman, J. (2019) Rotational Evolution of the Vela Pulsar during the 2016 Glitch.

[15] Hujeirat, A.A. and Thielemann, F.-K. (2009) $A \& A, 496,609$. https://doi.org/10.1051/0004-6361/200810793

[16] Camenzind, M. (2007) Compact Objects in Astrophysics. Springer, Heidelberg. 